\title{
"Estou sempre com sono, será doença?" - Caso clínico de narcolepsia na adolescência
}

\author{
"I'm always sleepy, am I ill?" - Case report of narcolepsy in adolescence
}

\author{
Joaquina Antunes', Pedro Fernandes ${ }^{1}$, Alzira Silveira ${ }^{2}$
}

${ }^{1}$ Internos Complementares de Pediatria, Serviço de Pediatria, Hospital de São Teotónio, Centro Hospitalar Tondela-Viseu, Viseu, Portugal; ${ }^{2}$ Especialista em Pediatria, Chefe do Serviço de Pediatria, Coordenadora da Unidade de Adolescência, Hospital de São Teotónio, Centro Hospitalar Tondela-Viseu, Viseu, Portugal.

\section{RESUMO}

Objetivos: A referência à sonolência diurna excessiva na adolescência é relativamente frequente e raramente tem uma causa patológica na sua origem. Os autores apresentam um caso de hipersonolência diurna devido à narcolepsia numa adolescente e fazem uma revisão da literatura sobre este distúrbio.

Descrição do Caso: Adolescente de 16 anos, sexo feminino, há um ano apresentando hipersonolência diurna e episódios de microssono durante suas atividades diárias, apesar de higiene de sono adequada. Exame objetivo sem particularidades. Estudo analítico, eletroencefalograma e estudo polissonográfico sem alterações. O teste de latências múltiplas do sono revelou uma latência média de 4:22 minutos e dois episódios de sono REM (Rapid Eye Movement) no início do sono (SOREMp), indicativos de hipersonolência grave com critérios de narcolepsia. Perante o diagnóstico iniciou tratamento com modafinil, com evolução favorável.

Conclusões: A narcolepsia infantil é uma patologia rara e subdiagnosticada. A doença tem repercussões negativas na esfera emocional, comportamental e escolar/profissional, que podem ser minimizadas por um diagnóstico e uma terapêutica adequados e oportunos.

DESCRITORES: PARASSONIAS DO SONO REM; DISTÚRBIOS DO SONO POR SONOLÊNCIA EXCESSIVA; NARCOLEPSIA; ADOLESCÊNCIA.

\section{ABSTRACT}

Aims: Excessive daytime sleepiness is relatively common in adolescence, and rarely has a pathological etiology. The authors report a case of excessive daytime sleepiness due to narcolepsy in a teenager, and a review of the literature about this disease.

Case Description: A 16 year-old adolescent, female, presented since one year ago with excessive daytime sleepiness and micro-sleeps during her daily activities, despite adequate sleep hygiene. Physical examination was normal. Analytical study, electroencephalogram and polysomnography unremarkable. The multiple sleep latency test revealed a sleep latency of 4:22 minutes and two REM (Rapid Eye Movement) periods at sleep onset (SOREMp), suggestive of severe hypersomnia with criteria of narcolepsy. Treatment with modafinil was started, with favorable outcome.

Conclusions: Narcolepsy is a rare and under diagnosed disorder in children. The disease has a negative impact on the child's emotional, behavioral and school/work life, which can be minimized by an adequate and timely diagnosis and therapeutics.

KEY WORDS: REM SLEEP PARASOMNIAS; DISORDERS OF EXCESSIVE SOMNOLENCE; NARCOLEPSY; ADOLESCENCE. 


\section{INTRODUÇÃO}

A referência à sonolência diurna excessiva (SDE) em consultas de adolescentes é relativamente frequente e raramente tem uma causa patológica. $\mathrm{O}$ sono é uma condição fisiológica envolvendo complexos mecanismos ao nível do sistema nervoso central e relacionando-se com diversos processos de desenvolvimento e maturação nos primeiros anos de vida, como funções homeostáticas para conservação de energia, reposição de neurotransmissores, remodelagem e modulação da sensibilidade de sinapses e recetores e consolidação de memória. É controlado por mecanismos cronobiológicos e homeostáticos (ritmo circadiano) que determinam a sua necessidade e frequência, definindo o ciclo sono-vigília. Este ritmo é regulado pela interação de fatores exógenos (alternância de período claro e escuro, horário escolar/laboral, etc.) e fatores endógenos (núcleo supraquiasmático do hipotálamo). ${ }^{1,2}$

O sono desempenha um papel importante no desenvolvimento físico e emocional, de forma particular na adolescência, período caracterizado por importantes mudanças biopsicossociais, cognitivas e comportamentais. O adolescente é um ser biologicamente programado para dormir e acordar mais tarde, dado que as mudanças biológicas e comportamentais que ocorrem nesse período condicionam um atraso de fase, resultando em menos horas de sono e consequentemente sonolência durante o dia. Além disso, o adolescente, sujeito a várias pressões sociais, tem muitas vezes um excesso de atividades que levam a que não durma adequadamente, tais como o uso excessivo de computador e telefone móvel, novos relacionamentos afetivos, saídas noturnas, etc. Este conjunto de fatores determina uma má higiene de sono e, consequentemente, o aparecimento de problemas de sono, que nessa faixa etária tem uma incidência entre os 14 e os $33 \% .^{2}$

Devido à intensa relação existente entre a qualidade do sono e a da vigília, o resultado mais imediato de um sono de má qualidade é a queda de rendimento no dia seguinte, com danos no período de vigília. No adolescente, estes passam por SDE, dificuldades de atenção e de concentração, lentidão de raciocínio e perda de memória, que conduzem a um mau desempenho escolar, flutuação de humor, ansiedade, baixa autoestima, problemas comportamentais, depressão, predisposição para acidentes, atraso no desenvolvimento pubertário, excesso de ganho ponderal e consumo de álcool e de substâncias psicoestimulantes. $^{2}$
Menos frequentemente, a SDE nessa faixa etária pode não ser devida a uma má higiene de sono, mas sim subjacente a um distúrbio que é importante diagnosticar e tratar adequadamente, para minimizar o impacto na vida do adolescente. Perante um adolescente com queixas de SDE, é importante uma história clínica completa direcionada para potenciais causas, com destaque para a história de sono (avaliar os ciclos sono/vigília, dificuldades em iniciar ou manter o sono, movimentos ou comportamentos anormais durante o sono), sintomas diurnos (sonolência, déficit de atenção, irritabilidade), frequência dos sintomas e evolução temporal, assim como um exame objetivo completo que inclua um exame neurológico. $\mathrm{O}$ diagnóstico diferencial da SDE devida a uma má higiene de sono deve ser feito com efeitos secundários de fármacos, narcolepsia, hipersonia idiopática, síndrome das pernas inquietas, apneia obstrutiva do sono e muitas outras patologias endócrinas e metabólicas. ${ }^{3}$

A narcolepsia é uma patologia neurológica rara e invalidante, caracterizada por uma desregulação dos limites entre o sono e a vigília. A prevalência mundial estimada é de 0,025 a $0,05 \%$, sendo mais frequente no Japão, sem predomínio de gênero. Os sintomas surgem mais frequentemente na segunda década de vida, podendo, no entanto, surgir logo na infância. ${ }^{4-8} \mathrm{~A}$ síndrome clínica da narcolepsia caracteriza-se por SDE, habitualmente acompanhada por cataplexia (episódios breves de perda ou diminuição do tônus muscular sem perda de consciência), e menos frequentemente por alucinações hipnopômpicas e/ou hipnagógicas e paralisia do sono. Esta tétrade clássica (sonolência patológica, cataplexia, alucinações e paralisia do sono) nem sempre está presente, principalmente no início da doença, o que não impede o diagnóstico. ${ }^{4,5}$

No presente artigo descrevemos o caso de uma adolescente referenciada à consulta por SDE que não era justificada por má higiene de sono, cuja investigação complementar indicou tratar-se de narcolepsia. Uma história clínica detalhada que não aponte para má higiene de sono e um exame objetivo que não identifique uma causa para um sono noturno insuficiente devem ser complementados com uma investigação de forma a procurar outras patologias associadas à SDE, que apesar de pouco frequentes, podem existir como neste caso.

\section{RELATO DO CASO}

Uma adolescente de 16 anos foi referenciada à Consulta de Adolescência (Ambulatório de Adolescência do Serviço de Pediatria do Hospital de São Teotó- 
nio, Viseu, Portugal) por sonolência diurna excessiva com cerca de um ano de evolução. O quadro caracterizava-se por adormecimento involuntário, de forma imperiosa, com microssonos reparadores no meio das suas atividades diárias. Esses episódios ocorriam várias vezes ao longo do dia, com duração variável, seguidos de despertares bruscos e de um período de vigília de boa qualidade. A ocorrência dos episódios verificava-se em diferentes contextos, na escola, em atividades com amigos, ou em casa. Negava sintomas sugestivos de cataplexia, alucinações hipnagógicas ou hipnopômpicas, ou paralisia do sono. Havia referência a uma higiene de sono adequada, com oito a nove horas de sono por noite, em que referia apenas "ranger de dentes". Negava insônia, respiração ruidosa, apneia do sono ou despertares noturnos, tal como outras parassonias, ou movimentos anômalos durante o período noturno.

$\mathrm{Na}$ avaliação biopsicossocial não revelou fatores de vulnerabilidade, não apresentando sintomas depressivos. Referia um bom ambiente familiar e uma adequada integração escolar, com bom aproveitamento. Tinha um grupo de amigos com idade semelhante à sua, com os quais costumava realizar atividades ao fim de semana e nas férias. Negava consumo de tabaco, álcool ou outras substâncias estupefacientes. Sem consumo habitual de nenhum fármaco.

Ao exame objetivo apresentava bom estado geral, sinais vitais sem alterações, com peso adequado à estatura (índice de massa corporal de $19,2 \mathrm{~kg} / \mathrm{m}^{2}$; percentil 15-50). O exame neurológico e o exame físico não apresentavam alterações. Antecedentes pessoais e familiares irrelevantes.

Foi realizada investigação complementar, cujo estudo analítico (hemograma, bioquímica, exame comum de urina, função tiroideia, pesquisa de drogas de abuso na urina) não revelou alterações. $\mathrm{O}$ eletroencefalograma com prova de sono mostrou um traçado dentro da normalidade, sem atividade paroxística.

$\mathrm{Na}$ polissonografia noturna (PSG) observou-se 467 minutos de tempo total de registro, com latência de sono de 47 minutos, latência ao sono REM (rapid eye movement) de 113 minutos e tempo total de sono (TTS) de 404 minutos (eficácia de sono de 86,5\%). O TTS distribui-se em cinco períodos de sono não REM (NREM)/REM, o último completo, com relação de tempos $13,2 \% \mathrm{R}, 7,3 \% \mathrm{~N} 1$ e $56,1 \% \mathrm{~N} 2$ e $23,4 \% \mathrm{~N} 3$. Não se verificaram eventos respiratórios, com um índice de apneia/hipopneias $0 / \mathrm{h}$ e um índice de despertar 7,9/hora. A saturação de oxigênio média foi de $98 \%$, com valor de CT90 de $0 \%$ e partindo de uma saturação basal de $90 \%$.
No teste de latências múltiplas do sono (TLMS) efetuado na manhã seguinte à PSG, duas horas após acordar, a paciente realizou quatro cochilos diurnos de 20 minutos, nos quais se observaram latências (t1 e t2) L1 5:30 minutos, L2 4:30 minutos, L3 5 minutos e L4 2:30 minutos, com uma latência média de 4:22 minutos. O número de SOREMp (sleep-onsent rapid eye movement periods: episódios de sono REM nos primeiros 15 minutos de sono) foi de dois em L1 e L3 (latência média de 3:15 minutos). Em face destas alterações, não houve necessidade de completar os cinco cochilos do teste (Figuras 1 e 2).

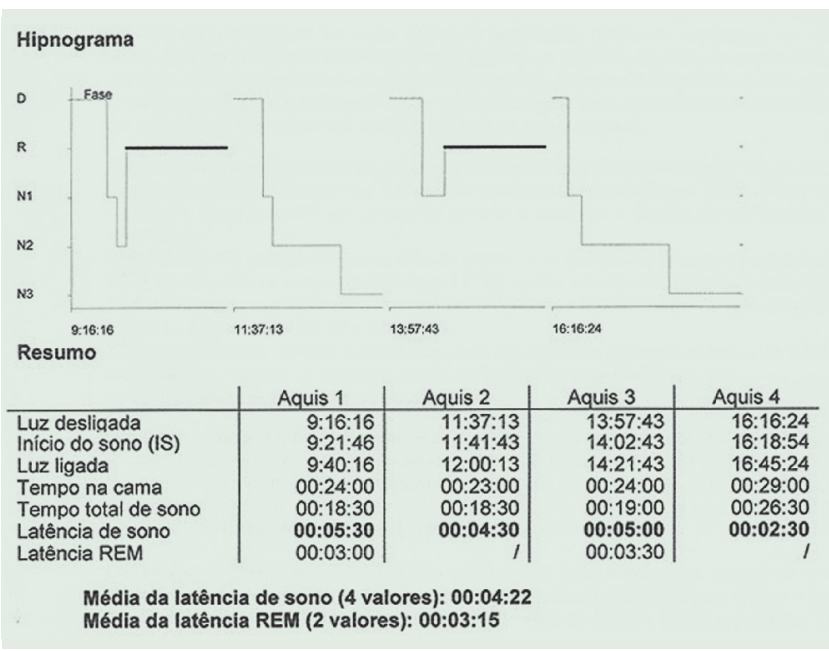

Figura 1. Hipnograma do teste de latências múltiplas do sono.

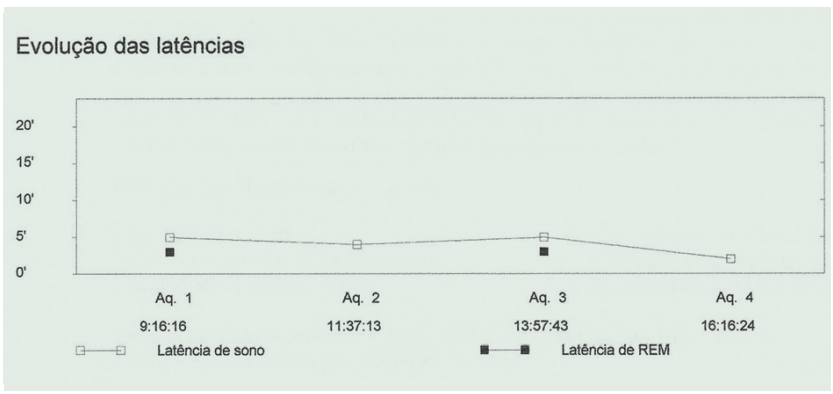

Figura 2. Evolução das latências ao longo do teste de latências múltiplas do sono.

A PSG permitiu verificar que a paciente apresentava um sono com diminuição do sono REM, além de insônia inicial e pequenos períodos de vigília intrassono que diminuíam a sua eficiência, o que poderia estar agravado pelo fato da paciente estar realizando o exame num ambiente estranho. A PSG excluiu alterações sugestivas de síndrome de apneia-hipopneia do sono ou de uma síndrome de resistência aumentada 
das vias aéreas. O TLMS indicou uma latência média patológica sugestiva de um processo de hipersonolência grave e dois episódios de sono REM nos primeiros 15 minutos de sono (SOREMp) o que pode estar relacionado com um processo de narcolepsia, uma vez excluídas outras causas de excessiva sonolência diurna secundária.

Perante a clínica e os exames complementares foi feito o diagnóstico de narcolepsia, sendo a adolescente encaminhada para consulta de patologia do sono. Iniciou terapêutica com modafinil $100 \mathrm{mg} /$ dia associada a medidas comportamentais, como realização de sestas programadas, verificando-se uma evolução favorável, com melhoria dos sintomas de SDE.

$\mathrm{Na}$ última avaliação, já com dois anos de seguimento, mantinha a terapêutica e apresentava-se clinicamente estável, sem referência a aparecimento de novos sintomas, encontrando-se bem integrada do ponto de vista social, escolar e familiar.

\section{DISCUSSÃO}

Narcolepsia é um termo de origem grega ("nárke" + "lepsis") que significa crise de sonolência ou apoderado pelo sono. É uma patologia neurológica crônica cada vez mais reconhecida, embora se considere que continua a ser subdiagnosticada, principalmente em idade pediátrica. Descrita pela primeira vez em 1877 pelo neurologista alemão Westphal como "um homem sofrendo de acessos súbitos de sono e episódios de inibição motora e da fala", foi denominada como tal três anos depois, em 1880, pelo francês Gélineau. ${ }^{4,6}$

A doença caracteriza-se pela presença de SDE, presente em todos os doentes, com necessidade imperiosa de sonos breves em situações ou lugares inapropriados, interrompendo qualquer tipo de atividade. Associa-se ainda a episódios breves de perda ou diminuição do tônus muscular sem perda de consciência, desencadeados por emoções como o riso e o medo (cataplexia). Podem estar associados outros sintomas menos frequentes, como as alucinações visuais, auditivas ou táteis no inicio do sono (hipnagógicas) e/ou na transição sono-vigília (hipnopômpicas) e a paralisia do sono. ${ }^{4-8}$

O início dos sintomas pode ocorrer da infância até a meia-idade, com dois picos de incidência aos $15 \mathrm{e}$ 35 anos. A maioria dos estudos disponíveis é referente a adultos, desconhecendo-se a prevalência exata em idade pediátrica. Existem, no entanto, alguns dados que apontam para que $34 \%$ dos doentes comecem com sintomas antes dos 15 anos, entretanto a variabilidade da apresentação atrasa o diagnóstico. ${ }^{4,9}$
Os principais sintomas nas crianças são semelhantes aos adultos, mas a sua manifestação pode ser diferente devido aos fatores maturativos. A SDE está sempre presente, embora em crianças menores os pais refiram apenas dificuldade em acordar de manhã ou a manutenção das sestas após os quatro anos de idade. A cataplexia é um sintoma comum em crianças, no entanto muitos só a desenvolvem anos depois de surgir a hipersonolência diurna, com uma progressão céfalo-caudal simétrica da debilidade muscular que pode até provocar queda no solo. Em um terço dos doentes temos apenas o status cataplégico atingindo os músculos da face ("face cataplegica") ou a protusão da língua associada ou não a movimentos lentos..$^{4,5,8}$

Nas crianças é comum a presença de um sono agitado com frequentes episódios de parassonias (terrores noturnos ou pesadelos). Como têm dificuldade em descrever as alucinações hipnagógicas/hipnopômpicas ou a paralisia do sono, devem ser sempre questionadas sobre a existência de sensações visuais estranhas. ${ }^{4,6}$

A doença em idade pediátrica pode ter comorbidades, como excesso de peso ou obesidade, que têm sido associadas aos níveis anormais de leptina e hipocretina e à diminuição do metabolismo basal, estando também descritos disartria, alterações da visão, ptose, cefaleias e puberdade precoce. Podem existir outros transtornos do sono (apneias, terrores noturnos, pesadelos, movimentos periódicos das extremidades ou transtorno do sono REM) e depressão. ${ }^{4,8}$

A etiologia da narcolepsia primária é desconhecida, mas os dados disponíveis apontam para a hipótese de uma doença neurodegenerativa com um componente autoimune que leva ao aparecimento da doença em indivíduos predispostos. $\mathrm{Na}$ maioria dos doentes temos a presença do antígeno de histocompatibilidade leucocitária (HLA) DQB1*0602, que também está presente em 38\% das crianças normais. Está também descrita a associação entre níveis baixos de hipocretina-1 no líquor e o desenvolvimento da doença. Valores inferiores a $110 \mathrm{pg} / \mathrm{ml}$ (30\% do valor médio) são muitos específicos de narcolepsia com cataplexia, enquanto valores superiores a $200 \mathrm{pg} / \mathrm{ml}$ são considerados normais. A diminuição do valor de hipocretina-1 no líquor é também descrita em outras doenças, como a síndrome de Guillain Barré e a encefalomielite aguda disseminada, pensandose que seja um processo autoimune o responsável pela destruição das células hipotalâmicas produtoras de hipocretina. A narcolepsia pode ser secundária a lesões tumorais, malformações vasculares, acidentes vasculares cerebrais, síndrome de Prader-Willi e Niemann Pick tipo C e outras síndromes genéticas. ${ }^{4,6,8,9}$ 
Quadro 1. Critérios diagnósticos de narcolepsia, com ou sem cataplexia, segundo a American Academy of Sleep Medicine (ICSD 2005).

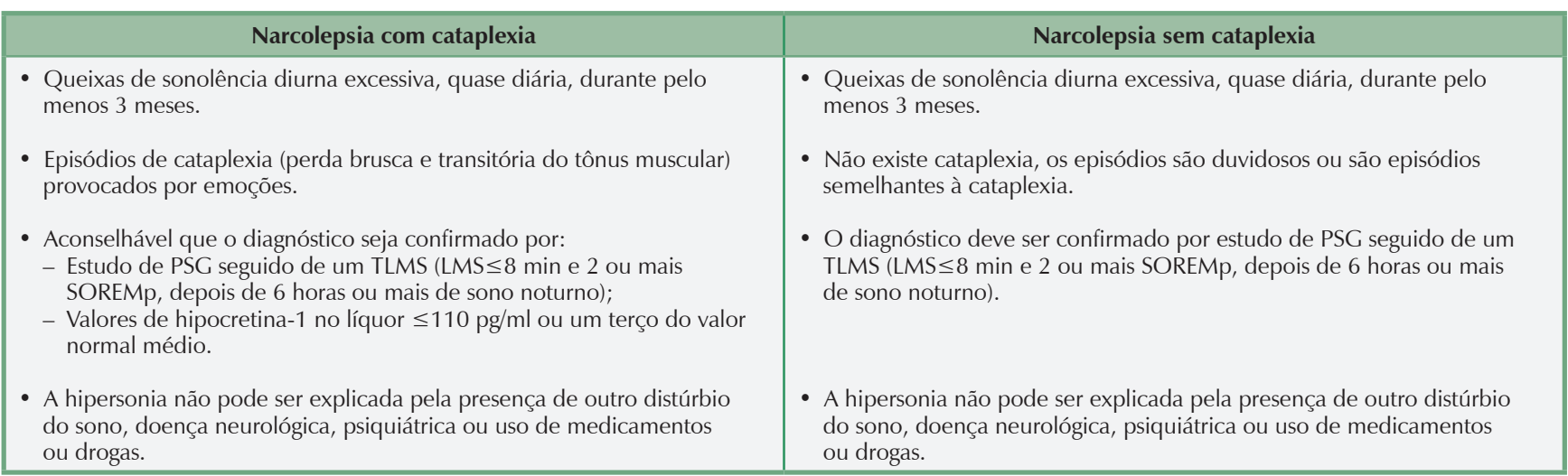

CSD: International Classification of Sleep Disorders; LMS: latências múltiplas do sono; PSG: polissonografia noturna; SOREMp: episódios de sono REM no início do sono (sleep onset REM periods); TLMS: teste de latências múltiplas do sono.

O diagnóstico da narcolepsia baseia-se na clínica (grau de suspeição), na PSG (exclusão de outros distúrbios; preferencialmente realizada pré TLMS) e TLMS. Os critérios de diagnóstico em idade pediátrica são os mesmos que para os adultos e foram definidos pela American Academy of Sleep Medicine (AASM) (Quadro 1). ${ }^{4-9}$

A PSG nos doentes com narcolepsia geralmente revela uma distribuição normal das fases do sono, podendo no entanto mostrar um aumento da percentagem do sono REM. A presença de episódios de apneiashipopneias ou movimentos periódicos dos membros, embora possa indicar outra etiologia para a sonolência diurna, não exclui o diagnóstico de narcolepsia, dado que essas perturbações do sono podem coexistir em alguns doentes. O TLMS constitui um método objetivo e validado, permitindo confirmar a presença de sonolência patológica. São apenas conhecidos valores normais acima dos oito anos, sendo estes semelhantes aos valores conhecidos nos adultos. Nestes, uma latência média do sono inferior a oito minutos e dois ou mais episódios de SOREMp são indicativos de narcolepsia. Em pediatria pode ser necessária a repetição desses testes ou a realização de outros, perante uma forte suspeita clínica com testes normais. ${ }^{4,6,8,9}$

Podem ser feitos também outros exames complementares, como a tipificação do HLA e a dosagem de hipocretina-1 no líquor, elementos importantes para o diagnóstico diferencial, não constituindo as suas alterações critérios de diagnóstico para a narcolepsia sem cataplexia. Sua realização é justificada nos casos com início muito precoce dos sintomas, se o quadro for grave ou se for impossível a realização de TLMS. A ressonância magnética cerebral deve ser realizada se houver sinais de gravidade, indícios de uma patologia subjacente ou se o exame neurológico estiver alterado. ${ }^{4,7,8}$

O padrão de início dos sintomas é muito variável, frequentemente insidioso, o que contribui para que este quadro seja pouco reconhecido pelos doentes, familiares e mesmo pelos médicos. É por vezes identificado apenas quando se associam sintomas de cataplexia, reconhecendo-se nessa altura que a criança sempre dormiu demasiado. ${ }^{4,6}$

O diagnóstico diferencial deve ser feito com epilepsia (especialmente crises atônicas), síncope neurocardiogênica, doenças psiquiátricas (transtornos conversivos, depressão grave, perturbação oposiçãodesafio, psicose) ou ingestão de tóxicos. Na adolescência é também importante excluir privação crônica do sono (síndrome de apneia-hipopneia, síndrome das pernas inquietas, má higiene de sono) ou transtornos do ritmo circadiano (síndrome de atraso de fase) como causas de sonolência diurna excessiva. ${ }^{4,8}$

Os problemas escolares são frequentes nestas crianças, sendo muitas vezes rotuladas como hiperativas e desatentas, devido à necessidade de serem mais irrequietas para combater a sonolência flutuante durante o dia, o que provoca inatenção e problemas de memória. Está também descrita a associação com sintomas depressivos, justificada pelo insucesso escolar e uma má aceitação entre pares, assim como a possibilidade de déficits de neurotransmissores, comum às duas entidades. ${ }^{4}$

O tratamento passa por uma abordagem integral dos aspectos biopsicossociais, envolvendo a criança, a família e a escola, associada ao tratamento farmamacológico. Um elemento fundamental para o sucesso do tratamento é a informação/esclarecimento e educação do paciente, dos familiares e dos professores 
acerca da doença. ${ }^{10}$ As medidas não farmacológicas incluem medidas comportamentais que promovam um estilo de vida saudável, respeitando uma boa higiene de sono com horários de sono-vigília regulares, e a prática de um desporto não violento, que também ajuda a combater a obesidade muitas vezes associada. Quando compatíveis com a vida escolar/profissional, a realização de breves sestas programadas, o consumo de bebidas ricas em cafeína e uma dieta pobre em hidratos de carbono têm demonstrado benefício no combate à sonolência diurna. $\mathrm{O}$ apoio psicológico pode ajudar nas situações de fraco suporte e incompreensão por terceiros..$^{4-6,10}$

Para tratamento farmacológico em idade pediátrica, estão aprovados pela Food and Drug Administration e por agências de medicamentos de outros países apenas os estimulantes do sistema nervoso central, sendo no entanto também utilizados outros fármacos que mostraram eficácia em adultos. O tratamento farmacológico inclui promotores da vigília (modafinil, dose inicial de $100 \mathrm{mg} / \mathrm{dia}$ ), psicoestimulantes (metilfenidato, dose mínima eficaz até ao máximo de
$60 \mathrm{mg} / \mathrm{dia}$ ), anfetaminas e mazindol como primeira linha. O oxibato de sódio tem demostrado eficácia em adultos, já estando também descrito o seu uso em idade pediátrica na narcolepsia com cataplexia, sendo necessários mais estudos que confirmem a sua eficácia e segurança nesta faixa etária. Em casos isolados está descrita uma resposta favorável aos imunossupressores e à imunoglobulina. Na cataplexia, embora sem indicação formal em menores de 18 anos, utilizam-se os antidepressivos tricíclicos (clomipramina, desimipramina, imipramina e protriptilina) e os inibidores da recaptação da serotonina e outras monoaminas (fluoxetina, venlafaxina, atomoxetina e reboxetina). ${ }^{4-6,10,11}$

A narcolepsia infantil constitui um problema de saúde com consequências importantes na esfera emocional, comportamental e com repercussão negativa a nível escolar. É importante suspeitar da doença perante qualquer criança/adolescente com SDE que não se justifique por outra causa, de forma a iniciar um plano terapêutico ajustado que minimize o impacto da doença na vida da criança/adolescente, tal como se verificou no caso descrito.

\section{REFERÊNCIAS}

1. Aloe F, Azevedo A. Mecanismos do ciclo sono-vigília. Rev Bras Psiquiatr. 2005;27(supl 1):33-9.

2. Ciampo LAD. O sono na adolescência. Adolesc Saude. 2012;9(2):60-6.

3. Wise MS, Glaze D. Assessment of sleep disorders in children. [Internet]. UptoDate Website [updated 2013; cited 2013 Aug]. Available from: http://www.uptodate.com

4. Merino-Andréu M, Martínez-Bermejo A. Narcolepsia con y sin Cataplejia: una enfermedad rara, limitante e infradiagnosticada. An Pediatr (Barc) 2009;71(6):524-34.

5. Mignot E. Genetic and familial aspects of narcolepsy. Neurology. 1998;50(2 Suppl 1):S16-22.

6. Cabral AS, Madeira N, Matos MJ, Ferreira AM. Narcolepsia: a propósito de um caso clínico. Rev Psiquiatr Cons Ligação 2006;2(13):29-37.

7. American Academy of Sleep Medicine. International Classification of Sleep Disorders. 2nd ed. Diagnostic and coding manual. Westchester: American Academy of Sleep Medicine; 2005.

8. Scammell TE. Clinical features and diagnosis of narcolepsy. [Internet]. UptoDate Website [updated 2013 june; cited 2013 Aug 10]. Available from: http://www.uptodate.com

9. Bozorg AM. Narcolepsy. [Internet]. Emedicine Website [updated 2013 march; cited 2013 Sept 10]. Available from: http://www.emedicine. medscape.com

10. Scammell TE. Treatment of narcolepsy. [Internet]. UptoDate Website [updated 2013; cited 2013 Aug 10]. Available from: http://www. uptodate.com

11. Wise MS, Arand DL, Auger RR, Brooks SN, Watson NF. Treatment of Narcolepsy and other Hypersomnias of Central Origin. SLEEP 2007;30(12):1712-27. 